\title{
Der neue MW-Herausgeber Prof. Dr. Georgios Gounalakis
}

\author{
Professur für Bürgerliches Recht, Internationales Privat- \\ recht, Rechtsvergleichung und Medienrecht, Institut für \\ Privatrechtsvergleichung am Fachbereich Rechtswissen- \\ schaften der Philipps-Universität Marburg
}

\section{Forschungsschwerpunkte der Professur}

Die Professur um Prof. Dr. Georgios Gounalakis forscht rechtsvergleichend und mit international privatrechtlichen Bezügen zu Fragen des Urheber- und Medienrechts, zum Recht des elektronischen Geschäftsverkehrs und zum Recht der Digitalisierung.

\section{Medienrecht}

Die Sicherung der Meinungsvielfalt ist dabei eine Kernaufgabe des Medienrechts. Die zunehmende Komplexität der medienökonomischen Gegebenheiten erschwert indes die rechtliche Umsetzung dieses an sich unbestrittenen Postulats. Untersucht wird der strukturelle Übergang vom Rundfunkkonzentrations- zum Medienkonzentrationsrecht, wobei auch das parallele Verfahren der Kartellbehörden zur Marktmacht-Kontrolle, insbesondere die Fusionskontrolle, in die Betrachtung einbezogen wird. Thematisiert werden die unterschiedlichen Teilaspekte der Vielfaltsicherung, indem die verfassungsrechtlichen Grundlagen ebenso erörtert werden wie die einfachgesetzliche Ausgestaltung im Rundfunkstaatsvertrag der Länder und deren Verhältnis zum allgemeinen Kartellrecht (Medienkonzentrationsrecht, Beck-Verlag).

Der Schutz der Persönlichkeit in den Medien ist ein weiterer zentraler Forschungsbereich: Die weltumspannende Kommunikation über das Internet macht Persönlichkeitsverletzungen zu einem globalen Risiko. Jedermann kann über das Netz kommunizieren, aber auch in seinen personalen Gütern verletzt werden: Beleidigungen in Chatrooms, Bilder auf Webseiten, Online-Archive, Bewertungsportale oder Namensanmaßungen durch unbefugten DomainGebrauch lassen den Persönlichkeitsschutz wichtiger denn je erscheinen. Deshalb ist es notwendig, die einzelnen Tatbestände und Rechtsbehelfe aufzuzeigen (Persönlichkeitsschutz im Internet, Grundlagen und Online-Spezifika, Beck-Verlag sowie Schutz der Persönlichkeit und Reputation in Online-Medien, in: Handbuch Persönlichkeitsrecht, Beck Verlag)
Eine rechtsvergleichende Betrachtung mit Länderberichten zu Deutschland, Frankreich, England und USA versucht Hinweise und Anregungen zu geben, wie das deutsche Recht des Persönlichkeitsschutzes verbessert werden kann (Privacy and the Media. A Comparative Perspective, Beck-Verlag).

\section{Urheberrecht}

Mit Einfügung des Urhebervertragsrechts in das Urheberrechtsgesetz können Urheber und ausübende Künstler unabhängig vom vertraglich vereinbarten Honorar im Nachhinein einen gesetzlichen Anspruch auf „angemessene Vergütung" erhalten. Das Gesetz stellt indes einen unverhältnismäßigen Eingriff in die verfassungsrechtlich geschützte Privatautonomie dar. Der Zwang zur kollektiven Regelung verstößt gegen Art. 9 GG. Auch missachtet der Entwurf das europäische Wettbewerbsrecht. Die Lücken des geltenden Rechts können mit weniger einschneidenden Regelungen geschlossen werden (Urhebervertragsrecht, Verfassungs- und europarechtliche Bewertung des Entwurfs der Bundesregierung vom 30. Mai 2001, VistasVerlag).

Mit $\S 52$ a Urheberrechtsgesetz kann es in den Bereichen Unterricht und Forschung ohne die Zustimmung des Urhebers bzw. Rechteinhabers in gewissem Umfang gestattet sein, urheberrechtlich geschützte Werke mit Hilfe elektronischer Datenübertragung zur Veranschaulichung im Unterricht oder zur eigenen wissenschaftlichen Forschung an Unterrichtsteilnehmer oder Wissenschaftler zu übermitteln (Stichwort Lehr- und Forscherplattformen). Als urheberrechtliche Schrankenregelung steht $\S 52 \mathrm{a}$ UrhG im Spannungsfeld der Verwertungsinteressen von Urhebern und Rechteinhabern einerseits und Interessen der Allgemeinheit an einer zustimmungsfreien Werknutzung andererseits. Ob es dem Gesetzgeber aber mit § 52a gelungen ist, den urheberrechtlichen Vermögensrechten im Verhältnis zur Privilegierung von Unterricht und Forschung in angemessener Weise Rechnung zu tragen, ist fraglich, 
wenn die Vergütung nicht gesichert ist (Elektronische Kopien für Unterricht und Forschung (§ 52a UrhG) im Lichte der Verfassung, Mohr-Verlag).

\section{Recht des elektronischen Geschäftsverkehrs}

Aus einer kaum mehr überschaubaren Fülle von Einzelaspekten zum Recht des elektronischen Geschäftsverkehrs wird versucht, wichtiges zu extrahieren, Überblicke zu verschaffen und spezifische Fragestellungen mit den allgemeinen rechtlichen Grundlagen zu verknüpfen. Einzelaspekte werden in den Gesamtkontext gestellt und zu einem Leitfaden gebündelt. Damit ist beabsichtigt, die relevanten Themenkomplexe für den Rechtsanwender verständlich zu machen, etwa zu zentralen Fragen: bei Geschäften der Service-Provider, bei Bank- und Versicherungsgeschäften, Internetauktionen, Elektronischen Informationsdiensten, Beratungsdienstleistungen, Telemedizin, Telearbeit, bei Music on Demand, Video on Demand, PayTV-Piraterie, beim Versandhandel, Softwarehandel, Internetfernsehen und Internetradio und zu vielem mehr. Alle Themengebiete werden unter nationalen und internationalen Aspekten beleuchtet, etwa zentrale EG-Richtlinien, wichtige ausländische Märkte wie USA, England, Frankreich, Niederlande, Österreich, Schweiz, Spanien, Australien und Süd-Korea sowie das internationale Privatrecht und die internationale Rechtsdurchsetzung (Rechtshandbuch Electronic Business, Beck-Verlag)

\section{Digitalisierung und Recht}

Schon länger wird am Institut zu Fragen der durch die Digitalisierung bedingten Veränderung rechtlicher Rahmenbedingungen geforscht. Begonnen hatten die Forschungsarbeiten bereits mit dem Gutachten für den Deutschen Juristentag 2002 zum Thema „Konvergenz der Medien - Sollte das Recht der Medien harmonisiert werden?" Die zunehmende Digitalisierung ermöglicht die Übermittlung immer verschiedenartigerer Dienste auf demselben Übertragungsweg und den Empfang mit einem einzigen Endgerät. Diese Entwicklung bedarf vor dem Hintergrund immer neuer Medien- und Kommunikationsdienste auch einer rechtlichen Ordnung und Regulierung. Die Rechtsordnung in Deutschland bietet hierbei ein überaus komplexes, jedoch wenig übersichtliches Regulierungssystem. Zentraler Orientierungsmaßstab für die fortschreitende Entwicklung sind die verfassungsrechtlichen Ausrichtungen und europarechtlichen Vorgaben. Mit dem Gutachten ist bereits versucht worden, hieran anzuknüpfen und die komplexen Fragen aufzuzeigen sowie Lösungsvorschläge zu entwickeln.

Die Digitalisierung hat in der Folge zu weiteren weitreichenden Veränderungen in der Medienlandschaft geführt, dem etwa das geltende Recht der Vielfaltsicherung nicht mehr

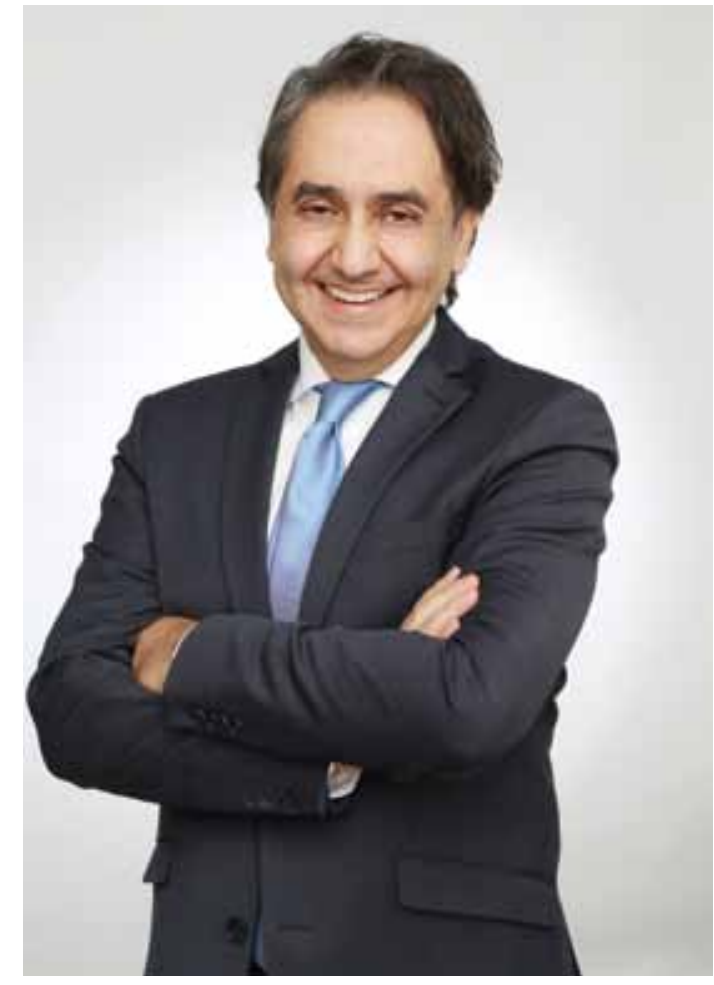

Prof. Dr. Georgios Gounalakis

gerecht wird. Dieser Prozess ist noch nicht abgeschlossen. In medienkonzentrationsrechtlicher Hinsicht sind die Auswirkungen auf die verschiedenen Medienmärkte Hörfunk, Presse, lineares und nichtlineares Fernsehen sowie online zu differenzieren. Eine Reform des Medienkonzentrationsrechts muss alle Teilmärkte in den Blick nehmen und sich am Gesamtmeinungsmarkt orientieren und nicht wie gegenwärtig, im Kern allein am Fernsehen.

Weitere Forschungsthemen im Bereich der Digitalisierung betreffen die Klärung von Rechtsfragen in Bezug auf

- Künstliche Intelligenz und Recht (Algorithmen, Bots etc.)

- Datenschutz im Zusammenhang mit der Datenschutzgrundverordnung

- Medienintermediäre wie etwa Suchmaschinen, Soziale Netzwerke, Instant-Messenger-Dienste, App-Plattformen etc.

- Digitale Medienplattformen mit ihrer Netzwerkstruktur, Datensammlung, Marktmacht und Veränderung der Kommunikation

- Kabelweiterleitung und urheberrechtliche Vergütung

- Funktionsauftrag und wirtschaftliche Betätigung des ZDF

- Elektronische Handelsplattformen für Unternehmen

- Wirtschaftliche Kooperation von Hörfunkanbietern

- Die Arbeit „Embryonenforschung und Menschenwürde“ ist zwischen Ethik und Recht verortet. 


\section{Service - In eigener Sache}

\section{Lehrschwerpunkte der Professur}

Das Bürgerliche Recht prägt neben dem Medienrecht und dem Internationalen Privatrecht die Lehre von Professor Gounalakis. Zudem beschäftigt sich die Professur in Seminaren mit rechtsvergleichenden urheber- und medienrechtlichen Fragestellungen.

Im Bürgerlichen Recht konzentriert sich die Lehre auf Vorlesungen zum Bürgerlichen Gesetzbuch (BGB). Im Fokus stehen vor allem der Allgemeine Teil des BGB, das Schuldrecht mit seinem Allgemeinen Teil und Besonderen Teil sowie auch das Sachenrecht. Hinzu kommen regelmäßig Übungen im Bürgerlichen Recht zur Propädeutik, für Anfänger sowie für Fortgeschrittene. Auch die Vorlesung zum Internationalen Privatrecht wird angeboten. Rechtsvergleichende Zusammenhänge werden vor allem in den medienrechtlichen Seminaren zu aktuellen Fragen des Urheberund Medienrechts behandelt. Die Vorlesung Medienrecht wird regelmäßig im Sommersemester im Rahmen des Schwerpunktbereichs angeboten.

\section{Internationale Kontakte und Bezüge zur Praxis}

Professor Gounalakis hat mehrfach Lehr- und Forschungsaufenthalte an ausländischen Universitäten wahrgenommen, zuletzt an der Monash University Melbourne/Australien. Daraus ist u.a. eine vergleichende Untersuchung australischer und deutscher Wissenschaftler zum Recht der Vielfaltsicherung im deutsch-australischen Vergleich entstanden (Media Diversity Law - Australia and Germany Compared, Lang-Verlag).

Professor Gounalakis ist als Erasmus- und Magisterbeauftragter des Fachbereichs zuständig für die Betreuung der ausländischen und deutschen Erasmus- und Magisterstudenten. Das Austauschprogramm, welches der Fachbereich unterhält, umfasst mehr als 30 Partneruniversitäten in Europa und Australien.

Kontakt zur Praxis hält Professor Gounalakis, indem er mit Rundfunkanstalten, Unternehmen, Verbänden, Kanzleien im Medienbereich und mit den Landesmedienanstalten kooperiert. Häufig war er als Gutachter gefragt und vielfach als Sachverständiger bei Anhörungen im Europäischen Parlament, im Bundestag und in verschiedenen Landtagen zu Fragen des Urheber- und Medienrechts tätig. Professor Gounalakis ist darüberhinaus Richter am Deutschen Medienschiedsgericht (DMS) sowie sachverständiges Mitglied der Kommission zur Ermittlung der Konzentration im Medienbereich (KEK), als deren Vorsitzender er fungiert. 


\section{Ausblick}

\section{Die MedienWirtschaft auf den Münchner Medientagen 2019}

Auch in diesem Jahr findet auf den Münchner Medientagen wieder ein Panel statt, das unsere Fachzeitschrift gemeinsam mit dem Bayerischen Rundfunk (BR) veranstaltet. Dieses BR-MW-Panel wird sich mit dem Thema

Verantwortung der Medien in der digitalen Welt befassen und ist wie folgt konzipiert:

\section{Müssen Medien Haltung zeigen? Verantwortung in der digitalen Welt}

Donnerstag, 24. Oktober 2019, 14:45 - 15:45 Uhr

Prof. Dr. Christoph Neuberger, Ludwig-Maximilians-Universität München,

Mitherausgeber der Fachzeitschrift MedienWirtschaft, Grußwort

Prof. Dr. Petra Grimm, Hochschule der Medien Stuttgart, Institut für Digitale Ethik,

Impulsvortrag: „Topografie der Digitalen Ethik“

Das Podium:

Dr. Susanne Pfab, Generalsekretärin der ARD

Angela Kesselring, Süddeutsche Zeitung, Leitung Kooperationsmanagement

Tina Kulow, Facebook Deutschland, Director Corporate Communications

Dr. Mark Schiffhauer, Zeit Verlagsgruppe, Chief Creative Officer

Moderation: Dr. Markus Riese, Bayerischer Rundfunk, Leiter Abteilung Marketing

Medienmarken nehmen vermehrt Stellung zu gesellschaftlichen Themen und bereichern damit den öffentlichen Diskurs. In diesem Diskurs werden das Selbstverständnis von Medienunternehmen zwischen einer Gewinnorientierung und einer Wertorientierung neu ausgehandelt.

Es genügt nicht mehr, dass das Unternehmen erfolgreich wirtschaften. Sie sollen auch deutlich einen wertvollen Beitrag zum Gemeinwohl - mit den Interessen nachfolgender Generationen im Blick - leisten.

Über die Erfüllung von Kundenwünschen hinaus geht es längst darum, individuelle Sinnbedürfnisse von Kunden zu erfüllen. Die digitalen Märkte werden durch diese Entwicklung zu einem weiteren Forum sozialer Orientierung und die Marken als Bedeutungsträger werden zu relevanten sozialen Akteuren. Die Grenze zwischen wertvollen Diskursbeiträgen und dominanten Marken müssen die Bürger als Konsumenten deshalb immer wieder neu bestimmen und damit den Marken in ihren Gesellschaften eine gemeinschaftsfördernde, kooperative Rolle zuweisen.

\section{Impressum}

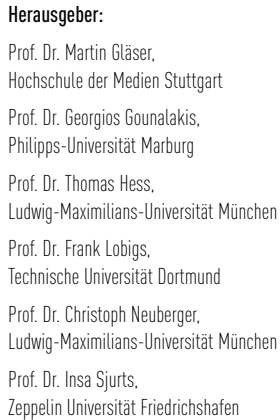

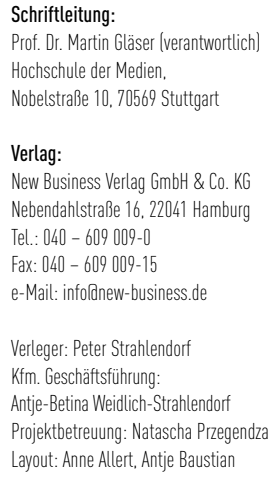

\section{Druck und Lithos:}

Lehmann Offsetdruck und Verlag GmbH Gutenbergring 39,22848 Norderstedt

\section{Bezugsbedingungen:}

Jahresabonnement 78, - Euro [zzgl. Versand und USt.).

Studentenabonnement 49,- Euro (zzgl. Versand und USt.).

\author{
Das Abonnement verlängert sich automatisch um ein \\ Jahr, wenn es nicht mit einer Frist von vier Wochen zum \\ Ende des Bezugszeitraumes schriftlich gekündigt wird. \\ Erscheinungsweise: 4-mal jährlich \\ Namentlich gekennzeichnete Artikel müssen nicht die \\ Meinung der Herausgeber/Redaktion wiedergeben. Un- \\ verlangt eingesandte Manuskripte - für die keine Haf- \\ tung übernommen wird - gelten als Veröffentlichungs- \\ vorschlag zu den Bedingungen des Verlages. Es werden \\ nur unveröffentlichte Orginalarbeiten angenommen. Die \\ Verfasser erklären sich mit einer nicht sinnentstellenden \\ redaktionellen Bearbeitung einverstanden. \\ ISSN 1613-0669
}




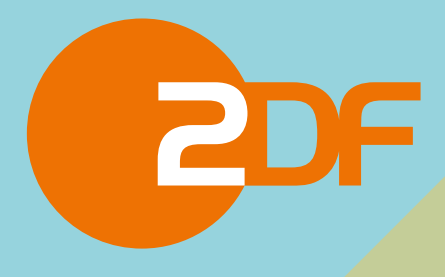

Hier ist Kultur

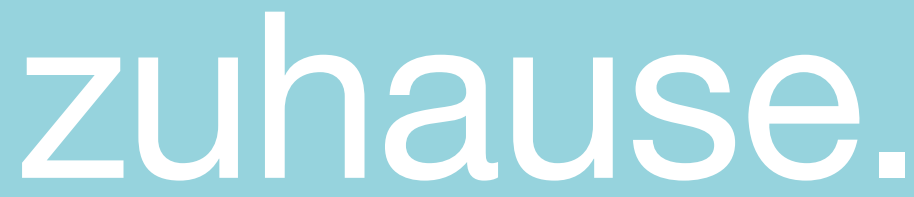

Mehr Infos unter zdfkultur.de

बिलिक 